\title{
Acute Iatrogenic Rhabdomyolisis and Guillan-Barre' Syndrome: A Dangerous Association
}

\author{
Lucia Briscese ${ }^{1}$, Elisa Giorli ${ }^{1,2}$, Tommaso Bocci ${ }^{1,2}$, Silvia Tognazzi ${ }^{1}$, Marta Seghieri ${ }^{3}$, Marco Taddei ${ }^{3}$ and Ferdinando Sartucci ${ }^{1,4,5 *}$
}

${ }^{1}$ Department of Neuroscience, AOUP, Pisa, Italy

${ }^{2}$ Department of Medical, Surgical and Neurological Sciences, University of Siena, Italy

${ }^{3}$ General Medicine II, Pisa University Medical School, Italy

${ }^{4} \mathrm{CNR}$ Neuroscience Institute, Pisa, Italy

${ }^{5}$ Department of Neuroscience, Pisa University Medical School, Pisa, Italy

\begin{abstract}
Guillan-Barré Syndrome (GBS) is an acute, symmetrical polyneuropathy with a clinical manifestation of flaccid paralysis with areflexia and variable sensory disturbance. GBS has an incidence of 1-2 cases/100.000 inhabitants for year. The pathological spectrum of GBS includes Acute Inflammatory Demyelinating Polyneuropathy (AIDP), Acute Motor Axonal Neuropathy (AMAN) and Acute Motor Sensory Axonal Neuropathy (AMSAN). We report a case of an 81-year-old man with GBS (subtype AMSAN), secondary to a previous Micoplasma Pneumoniae infection, who presented with an elevation of Creatin Kinase (CK) serum levels, and worsened by a co-administration of statins and clarithromycin. By our knowledge there are few cases in the literature in which the association of these drugs contributed to worsening GBS.
\end{abstract}

Keywords: Guillan-Barré syndrome; Simvastatin; Clarithromycin; Rhabdomyolysis; AMSAN

\section{Introduction}

GBS is an acute demyelinating disease of peripheral nerves, which usually follows viral or bacterial illness. There is considerable evidence that GBS is an immune-mediated disease often due to a gastrointestinal or, more rarely, to a lung infection [1]. However, the exact pathogenesis remains unclear. In this case report, we describe a patient in which a preceding lung infection, associated with a co-administration of simvastatin and clarithromycin, resulting in rhabdomyolysis and acute inflammatory demyelinating polineuropathy.

\section{Case Report}

An 81-year-old Caucasian man presented to the emergency department complaining of dyspnea, chest and abdominal pain and severe and progressive weakness for the last 30 days.

His medical history was significant for hypertension, BPCO, diabetes type 2, gout, mild renal failure, heart failure after myocardial infarction (ejection fraction 54\%) and coronary heart disease. Two weeks before admission in our department, the same patient had been admitted to for dyspnoea, chest pain and elevated temperature. This clinical condition has due to pneumonia, and was treated with clarithromycin $500 \mathrm{mg}$ twice a day. He had been taking, among other drugs, simvastatin $20 \mathrm{mg} /$ once/day for almost one year before.

The patient, after 14 days of discharge returned to the hospital, with classical signs of rhabdomyolisis. Laboratory chemistry revealed a significant elevation of CK (maximum dosage detected $3956 \mathrm{U} / \mathrm{l}$; normal range 60-190 UI/l), myoglobin (maximum $5049 \mathrm{ng} / \mathrm{ml}$; normal range $\leq 90 \mathrm{ng} / \mathrm{ml}$ ) and myoglobinuria (maximum $1010 \mathrm{mg} / \mathrm{L}$; normal range $4 \mathrm{mg} / \mathrm{L}$ ); also GPT-ALT (maximum $142 \mathrm{UI} / \mathrm{l}$; normal range 9-63 UI/l), GOT-AST (maximum $77 \mathrm{U} / 1$; normal range 13-41 UI/L) and GGT (maximum 175U/l; normal value $\leq 55 \mathrm{U} / \mathrm{L}$ ) levels were raised. Neurological examination showed a moderate tetraparesis, muscles very painful to pressure, and tendon reflexes unremarkable in the upper extremities; instead, in the legs, tendon reflexes could not be elicited. Needle electromiography (EMG) did not reveal a typical myopathic pattern, but during the routine F-wave evaluation we recorded the presence of A-wave. The electroneurography displayed a motor-sensitive predominantly axonal poliradicoloneuropathy. The examination of cerebro-spinal fluid (CSF) showed a moderate damage of emotion-encephalic barrier with an albumin-citologic dissociation and systemic production of 6 oligoclonal immunoglobulins. The simvastatin was stopped and treatment with forced urine alkalization was started. A diagnosis of GBS syndrome was made and the patient was started intravenous immunoglobulins therapy with prompt improvement: the paresis improved and the serum CK and myoglobin dropped to normal levels ( $<159 \mathrm{U} / \mathrm{l}$ and $<85 \mathrm{ng} / \mathrm{ml}$ respectively).

\section{Discussion}

Usually, one-half to two-third of GBS patients report antecedent infections, and Campylobacter jejuni, Cytomegalovirus (CMV), Epstein-Barr virus (EBV) and Mycoplasma pneumonia are recognised triggering agents of GBS [2]. Moreover, Micoplasma Pneumoniae infection appears to be associated strongly with GBS cases (15\%) [3]. Moreover, marked raised levels of CK has been recognized and documented in GBS, correlating with occurrence of pain, [4] which was a presenting symptom in this patient. Raised level of CK can be a feature of the early course of GBS, and may cause diagnostic confusion with other conditions, particularly if the clinical picture is not typical. In fact, rhabdomyolysis is also a rare side-effect of 3-hydroxy-3-methylglutaryl coenzyme A (HMGA-CoA) reductase inhibitors. Co-medication with inhibitors of the CYP34A pathway-as Clarithromycin- may increase this risk of rhabdomyolysis [5]. In our case, the patient, 3 weeks before

*Corresponding author: Ferdinando Sartucci, Neuroscience Department, Pisa University Medical School, Pisa (Italy); Tel: +39.050.992176; Fax: +39.050.550563; E-mail: F.Sartucci@neuro.med.unipi.it

Received December 14, 2012; Accepted December 18, 2012; Published December 20, 2012

Citation: Briscese L, Giorli E, Bocci T, Tognazzi S, Seghieri M, et al. (2013) Acute latrogenic Rhabdomyolisis and Guillan-Barre' Syndrome: A Dangerous Association. J Neurol Neurophysiol 4:141. doi:10.4172/2155-9562.1000141

Copyright: (c) 2013 Briscese L, et al. This is an open-access article distributed under the terms of the Creative Commons Attribution License, which permits unrestricted use, distribution, and reproduction in any medium, provided the original author and source are credited. 
Citation: Briscese L, Giorli E, Bocci T, Tognazzi S, Seghieri M, et al. (2013) Acute latrogenic Rhabdomyolisis and Guillan-Barre' Syndrome: A Dangerous Association. J Neurol Neurophysiol 4:141. doi:10.4172/2155-9562.1000141

the first admission to the hospital, had presented dyspnea and chest pain, so atypical pneumonia was suspected and diagnosis of pneumonia was made based with clinical and instrumental texts (including chest X-ray (Figure 1), High Resolution Computer Tomography (HRCT) (Figure 2), tracheo-bronchial endoscopy with bronchial aspirated for cytologic and bacteriological examination). In addition, our patient started having symptoms of increased fatigue, shortness of breath, progressive and severe diffuse myalgia, cramps, exercise intolerance weakness, functional disability (inability to raise arms and legs) with the beginning of clarithromycin therapy. Clarithromycin, has been implicated in other many reports of statin-induced rhabdomyolysis $[6,7]$ but was used only after signs and symptoms had developed. In the literature several reports show an important rhabdomyolysis following the co-administration of statins and macrolide antibiotics (such as clarithromycin or erythromycin), or other drugs that inhibit the cytochrome CYP3A4 pathway, but most of patients described in these studies were treated with high doses of statins (i.e. simvastatin $80 \mathrm{mg} / \mathrm{die}$ ). Our case demonstrates that even low-dose therapy with statins may result in severe side effects, when combined with CYP3A4-inhibiting drugs, especially in patients with kidney failure. Moreover, the previous pneumoniae could be triggering the GBS that was exacerbated by the use of dangerous concurrent medication with simvastatin and clarithromycin. In fact, the electromiographic pattern as considered the presence of rhabdomyolysis, but it was consistent with an AMSAN. The EMG in fact showed a nerve damage with a widespread denervation activity in progress on both left and right tibial anterior muscles, on the right side of the diaphragm and on the I first dorsal interosseous, suggestive of a motor-sensitive predominantly axonal poliradicoloneuropathy (Figure 3).

In addition to neurophysiological examinations, that confirm this clinical picture, we have the result of CSF's analysis. From a simple point of view, a sudden muscle denervation could account for release of muscle enzymes in acute axonal poliradiculoneuropathies, up to seven times of the upper limit of normal; however, in a previous report Scott and colleagues [8] highlighted a relative sparing of small autonomic fibres in early phases of illness in a patient presenting with acute rhabdomyolysis and later developing an atypical Guillain-Barrè axonal neuropathy. That makes unlikely an elevation of muscle enzymes as epiphenomena of severe denervation in progress.

\section{Conclusion}

In conclusion, by our knowledge, this is the first case in which GBS, due to a preceding lung infection, was strongly exacerbated by a severe rhabdomyiolysis for a co-administration of simvastatin at low

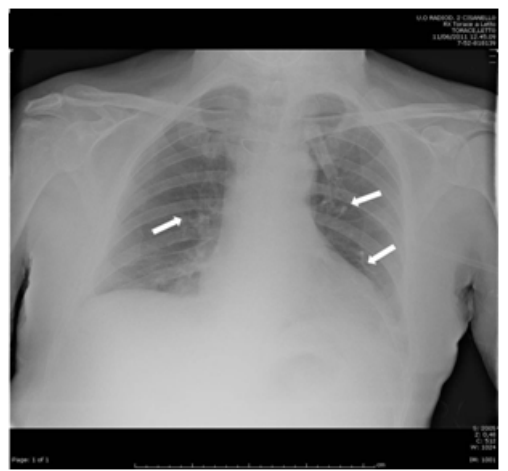

Figure 1: Chest X-ray. Consolidation in the right emithorax and interstitial spread thickening.

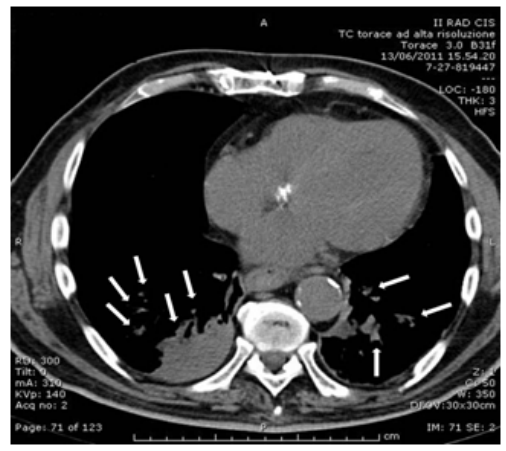

Figure 2: High Resolution Computer Tomography (HRCT). Inflammatoryflogistic finding. Note the thickening of bronchial walls, the signs of phlegmatic infarction and soft mass with nodular and ramified morphaology.

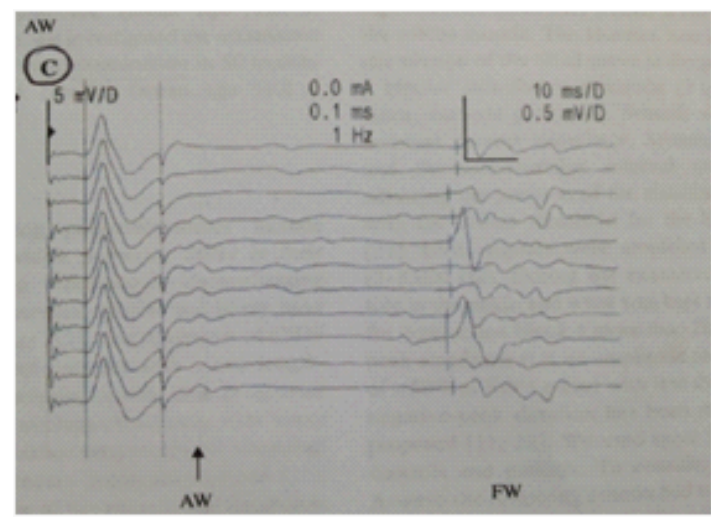

Figure 3: A-waves records. Set of A-waves recorded in our case of acute form of plyradiculoneuropathy.

dose, and clarithromycin. Moreover, our case shows how is important to evaluate the use of statins in association with others CYP3A4 (Cytochrome P450 3A4) inhibiting drugs, particularly in patients with prior infective conditions, renal insufficiency or other risk factors such as diabetes, heart failure, age $>80$ years. It is crucial, in particular among physicians, to have an awareness of the interaction's risk associated with these conditions because the early diagnosis and aggressive therapy may reduce the comorbidity and mortality and could result in rapid improvement, as shown in the present patient. In particular it is important to make an early diagnosis, through the use of laboratory tests and neurophysiological tests, and it's also important to know the medical history of the patient and his treatment history to suspend or avoid, in case of suspected rhabdomyolysis, any dangerous associations.

\section{References}

1. Pritchard J, Hughes RA (2004) Guillain-Barré syndrome. Lancet 363: 2186 2188.

2. Hadden RD, Karch $\mathrm{H}$, Hartung HP, Zielasek J, Weissbrich B, et al. (2001) Preceding infections, immune factors, and outcome in Guillain-Barré syndrome. Neurology 56: 758-765

3. S Sinha, KN Prasad, D Jain, CM Pandey, S.Jha (2007) Preceding infections and anti-ganglioside antibodies in patients with Guillan-Barré syndrome: a single centre prospective case control study, Clinical Microbiology and infection. Clin Microbiol Infect 2007: 334-337.

4. Ropper AH, Shahani BT (1984) Pain in Guillain-Barré syndrome. Arch Neurol 41: $511-514$

5. Wagner J, Suessmair C, Pfister HW (2009) Rhabdomyolysis caused by comedication with simvastatin and clarithromycin. J Neurol 256: 1182-1183. 
Citation: Briscese L, Giorli E, Bocci T, Tognazzi S, Seghieri M, et al. (2013) Acute latrogenic Rhabdomyolisis and Guillan-Barre' Syndrome: A Dangerous Association. J Neurol Neurophysiol 4:141. doi:10.4172/2155-9562.1000141

Page 3 of 3

6. Schreiber DH, Anderson TR (2006) Statin-induced rhabdomyolysis. J Emerg Med. 2006: 177-180.

7. Molden E, Andersson KS (2007) Simvastatin-associated rhabdomyolysis after coadministration of macrolide antibiotics in two patients. Pharmacotherapy 27: 603-607
8. Scott AJ, Duncan R, Henderson L, Jamal GA, Kennedy PG (1991) Acute rhabdomyolysis associated with atypical Guillain-Barré syndrome. Postgrad Med J 67: 73-74 